\title{
Supporting shared decision-making for older people with multiple health and social care needs: a protocol for a realist synthesis to inform integrated care models
}

\author{
Frances Bunn, ${ }^{1}$ Claire Goodman, ${ }^{1}$ Jill Manthorpe, ${ }^{2}$ Marie-Anne Durand, ${ }^{3}$ \\ Isabel Hodkinson, ${ }^{4}$ Greta Rait, ${ }^{5}$ Paul Millac, ${ }^{6}$ Sue L Davies, ${ }^{1}$ Bridget Russell, ${ }^{1}$ \\ Patricia Wilson ${ }^{7}$
}

To cite: Bunn F, Goodman C, Manthorpe J, et al. Supporting shared decisionmaking for older people with multiple health and social care needs: a protocol for a realist synthesis to inform integrated care models. BMJ Open 2017;7:e014026. doi:10.1136/bmjopen-2016014026

- Prepublication history for this paper is available online. To view these files please visit the journal online (http://dx.doi.org/10.1136/ bmjopen-2016-014026)

Received 25 August 2016 Revised 7 November 2016 Accepted 11 January 2017

CrossMark

For numbered affiliations see end of article.

Correspondence to Dr Frances Bunn; f.bunn@herts.ac.uk

\section{ABSTRACT}

Introduction: Including the patient or user perspective is a central organising principle of integrated care. Moreover, there is increasing recognition of the importance of strengthening relationships among patients, carers and practitioners, particularly for individuals receiving substantial health and care support, such as those with long-term or multiple conditions. The overall aims of this synthesis are to provide a context-relevant understanding of how models to facilitate shared decision-making (SDM) might work for older people with multiple health and care needs, and how they might be applied to integrated care models.

Methods and analysis: The synthesis draws on the principles of realist inquiry, to explain how, in what contexts and for whom, interventions that aim to strengthen SDM among older patients, carers and practitioners are effective. We will use an iterative, stakeholder-driven, three-phase approach. Phase 1: development of programme theory/theories that will be tested through a first scoping of the literature and consultation with key stakeholder groups; phase 2: systematic searches of the evidence to test and develop the theories identified in phase 1; phase 3: validation of programme theory/theories with a purposive sample of participants from phase 1. The synthesis will draw on prevailing theories such as candidacy, self-efficacy, personalisation and coproduction.

Ethics and dissemination: Ethics approval for the stakeholder interviews was obtained from the University of Hertfordshire ECDA (Ethics Committee with Delegated Authority), reference number HSK/SF/ $\mathrm{UH} / 02387$. The propositions arising from this review will be used to develop recommendations about how to tailor SDM interventions to older people with complex health and social care needs in an integrated care setting.

\section{BACKGROUND AND RATIONALE}

Navigating health and social care systems is particularly difficult for older people with

\section{Strengths and limitations of this study}

Shared decision-making (SDM) is a policy priority and a central organising principle of integrated care, in the UK and elsewhere.

- Although there is evidence that SDM can improve patient satisfaction and self-care skills, there is a lack of evidence about how to consistently and effectively implement SDM across health and care settings, particularly for older people with complex health needs.

- The synthesis draws on the principles of realist inquiry, to explain how, in what contexts and for whom, interventions that aim to strengthen SDM among older patients, carers and practitioners are thought to work.

- The propositions arising from this review will be used to develop recommendations about how to tailor SDM interventions to older people with complex health and social care needs in an integrated care setting

complex health needs, including those with dementia, frailty and multimorbidity, ${ }^{1}$ and they are at particular risk of poor continuity and fragmentation of care. ${ }^{23}$ Integrated care aims to address these problems, prevent duplication of services and reduce costs. ${ }^{4} 5$ In England, the 5-Year Forward View set out new models of integrated care as part of the strategic plan for wider system change in the National Health Service (NHS). ${ }^{6}$ These models have been piloted in 37 vanguard sites whose brief is to address traditional divides between primary care, community services and hospitals, and achieve personalised and coordinated healthcare through better integration. ${ }^{6-9}$

Shared decision-making $(\mathrm{SDM})$ is a process in which health and social care practitioners and patients work together to select 
tests, treatments, management or support packages of care and/or consider safeguarding outcomes, that are aligned with the patient's informed preferences. ${ }^{10} 11$ SDM is seen as a central organising principle of integrated care. ${ }^{4512}$ However, although there is evidence that SDM, and the use of patient decision aids, can improve knowledge, risk perception, patient participation in decision-making and self-management, ${ }^{13}$ there is a lack of evidence about how to successfully and consistently implement SDM across organisations and in routine healthcare settings. ${ }^{14} 15$

SDM may be particularly difficult in integrated care sites where decision-making and communication need to be negotiated among, and communicated to, multiple health and social care practitioners, including personal assistants directly employed by care users, as well as patients and their family carers. Moreover, for those most reliant on health and social care support, such as people who are very frail and those with severe disabilities arising from long-term conditions, decision-making may be particularly complex involving matters such as resource availability, polypharmacy, consent, concordance, the capacity of patients to attend to healthcare demands, support networks, safeguarding and the appropriateness of treatment in people with multimorbidity. ${ }^{16-18}$ Furthermore, depression is common in people with long-term conditions ${ }^{19}$ and may impact negatively on relationship building and engagement in SDM. The skills required for sharing personalised information with this vulnerable group, and with their family carers, can be hard to embed in services.

Older people with complex health and care needs are often reliant on others, typically family members, to advocate or negotiate access to care on their behalf. ${ }^{17}$ 20-22 Models, such as the triangle of care and patient-centred approaches, recognise that families are often crucial allies for quality and safety and should, subject to patient agreement, be routinely involved in decision-making for older people with complex health needs $^{23-25}$ or act as proxies where decision-making ability is severely compromised. Recent research has found, however, that although service providers recognise the contribution of family carers to the coordination and management of care, this does not translate into routine engagement of family carers in decision-making. ${ }^{17}$

Models of care that acknowledge the impact of this complexity include those that promote the individualisation or simplification of treatment, for example, for older people with diabetes, ${ }^{26}{ }^{27}$ and those that recognise the need to consider the ability of patients and their family carers to attend to healthcare demands. ${ }^{18} 28$ Minimally disruptive medicine is a theory-based approach that is focused on achieving patient goals for life and health while imposing the smallest possible burden on patients' lives. ${ }^{18}{ }^{29}$ Such approaches, however, require mechanisms or models of care that can facilitate relationships, meaningful discussion and SDM among a range of different providers, patients and carers. $^{30}$

To develop a theoretical understanding of the realities of working in and across complex, overlapping systems of care, and why and how different interventions to promote SDM may work, there is a need to synthesise the different strands of research evidence. Realist synthesis methodology will enable us to deconstruct the component theories underpinning different interventions aimed at promoting SDM with older people with complex needs. It will enable us to consider relevant contextual data to test our understanding of the applicability of different approaches for this population and how SDM might achieve desired outcomes, such as improvements in patient safety, clinical effectiveness, quality of life and optimal patient experience, ${ }^{31}$ within the context of integrated care.

\section{Aim and objectives}

The overall aims of the synthesis are to:

1. Identify key features or mechanisms within different programmes and approaches that have the potential to strengthen relationships between older people with multiple health and care needs, their family carers, and community health and social care providers;

2. Provide a context-relevant understanding of how models to facilitate SDM might work for older people with multiple health and care needs, and how they might be used to facilitate person-centred care in collaborative models of health and social care.

\section{The objectives are to}

1. identify how interventions, or elements of interventions, to promote SDM with older people with multiple long-term health and social care needs, and their family carers, are thought to work, on what outcomes and for whom they work (or why they do not work);

2. explore how models to facilitate SDM with older people with multiple health and social care needs might be incorporated into service delivery in collaborative models of care in order to achieve outcomes that reflect person-centred care;

3. explore how different contexts support or inhibit stakeholders' responses to activities that support SDM in collaborative care models;

4. inform the choice of process and outcome measures to assess the impact of SDM and person-centred care in the English NHS vanguard sites;

5. identify areas for future research, including promising interventions that merit further evaluation.

\section{METHODS}

Methodological approach

Realist synthesis is a systematic, iterative, theory-driven approach designed to make sense of diverse evidence 
about complex interventions applied in different settings. ${ }^{32-35}$ A realist synthesis takes a 'generative' approach to causation, that is, 'to infer a causal outcome $(\mathrm{O})$ between two events ( $\mathrm{X}$ and $\mathrm{Y}$ ), one needs to understand the underlying mechanism (M) that connects them and the context $(\mathrm{C})$ in which the relationship occurs' ${ }^{36}$ Realist synthesis is typically used to understand complex interventions which 'often have multiple components (which interact in non-linear ways) and outcomes (some intended and some not) and long pathways to the desired outcome(s) ${ }^{35}$ Central to the realist review process is the development of programme theory, that is, what a programme or intervention comprises and how it is expected to work. ${ }^{35}$ The reporting of the review will be guided by the Realist and Meta-narrative Evidence Syntheses: Evolving Standards (RAMESES) criteria for realist review ${ }^{35}$ International prospective register of systematic reviews ((PROSPERO) 2016 registration number: CRD42016039013).

The synthesis will focus on community-dwelling older people ( $\geq 65$ years) with complex health and care needs, for example, people with frailty, multimorbidity, longterm conditions, dementia and those who require help with personal care. The rationale for focusing on this group is that they usually have experience of using many health and social care services, their needs change over time and/or suddenly, often with progressive loss of cognitive and/or physical function, a family carer is frequently involved in their care, and they are often at risk of exacerbation of their illness, ${ }^{16}$ and death. In addition, many find it difficult to navigate complicated and underresourced services and are particularly vulnerable to fragmented care. ${ }^{2}$

While there is not a single intervention that constitutes SDM, it is a concept which is characterised by a set of assumptions or values. These relate to a belief in the rights of individuals to self-determination and patientcentred care, the use of evidence to guide decisions and reduce inappropriate clinical treatment and the need for mechanisms to deal with clinical uncertainty. ${ }^{37-39}$

\section{Research plan}

We are undertaking a three-phased approach that optimises the knowledge and networks of the research team. Stakeholders are important in realist work and the realist synthesis focus is driven by 'negotiation between stakeholders and reviewers'. ${ }^{34}$ The assumption of this research is that a realist review on interventions to promote SDM has to consider a range of theoretical frameworks. This is likely to include theories around the following:

- agency, advocacy and candidacy and how they may impact on access to care for vulnerable groups; ${ }^{22}$

- the role of individual-related and system-related factors in the development of interprofessional models of SDM; ${ }^{40} 41$

- minimally disruptive medicine and complex adaptive systems; ${ }^{18}$
- shared or proxy decision-making relevant to vulnerable groups ${ }^{42}$ who may lack decision-making capacity;

- the involvement of older people and their family members in their health and care, extending to coconstruction or coproduction theory; ${ }^{43}$

- the role of technology in the involvement of patients and carers in their care, for example, patient-held records, patient portals; ${ }^{43-45}$

- the 'expert patient' and self-management of longterm conditions; ${ }^{46} 47$

- prognostic framing of death and dying and how this can shape preferences and choices; ${ }^{48} 49$

- promoting continuity of care for older people, ${ }^{50} 51$ and the role of personal budgets or family carers in fostering continuity. ${ }^{17}$

\section{Phase 1: defining scope and developing programme theories}

In phase 1, we will develop programme theory/theories or hypotheses about why programmes that seek to promote SDM do, or do not, work. We will scope the literature to identify: existing theories on how and why the involvement of patients and carers are thought to be important, how they are defined in the literature, how interventions to promote SDM are meant to work and on which outcomes and how SDM might work in interprofessional settings. We will search Pubmed, The Cochrane Library and Google Scholar concentrating, in the first instance, on identifying systematic reviews of SDM. Search terms will include the following: 'decision aid', 'decision support', 'patient decision making', 'patient participation', 'shared decision making', 'proxy decision making', 'collaborative care', 'co production' and minimally disruptive medicine; combined with terms to identify systematic reviews. Additional searches will be run using the terms (multidisciplinary OR interdisciplinary) AND shared decision-making.

In addition, we will consult with up to 20 representatives from the following stakeholder groups:

- commissioners and service providers from organisations initiating integrated care-including three of the vanguard sites (Tower Hamlets Integrated Provider Partnership, East and North Herts Clinical Commissioning Group and Whitstable MCP) and with South Kent Coast Integrated Care Organisation $(\mathrm{n}=5)$;

- providers of health and social care in community settings involved in initiatives to involve patients and their family carers in their care-for example, GPs, practice nurses, community nurses, occupational therapists and social care managers $(n=5)$;

- older people and family carers who have experience of multiple practitioners and services-recruited from the UH Patient and Public Involvement in Research Group (PIRG) and Social Care Workforce Research Unit's User and Carer Group that includes older people who are frail, and carers from diverse backgrounds $(n=5)$; 
- advocacy and user/carer groups such as Age UK London, Carers UK, National Voices (http://www. nationalvoices.org.uk/coordinated-care), Healthwatch and Greater London Forum for Older People. Particular attention will be paid to the recruitment of people from black and minority ethnic groups and other under-represented groups $(\mathrm{n}=5)$.

Consultation will be done via individual face-to-face or telephonic interviews. However, our experience is that sometimes older people request that a family member is present in any interview and, in such instances, we will abide by their preferences. The purpose of the consultation with stakeholders will be to explore key assumptions about what needs to be in place for effective SDM within integrated care initiatives, identify relevant outcomes and clarify the focus and scope of the searches in phase 2 . Interviews will be guided by a topic guide which will be used to explore understanding and experiences of SDM, including what works well and what could be improved. Data from the interviews will be regularly reviewed in order to ensure that the topic guide remains fit for purpose and to assess whether saturation has been reached. If participants find it difficult to talk about SDM, then the team will use vignettes as prompts for discussion. Vignettes will be developed via facilitated discussion that draws on the collective research and clinical experience of the project team. They will involve a variety of circumstances and social actors (eg, different health professionals, the older person and family carers), and will be verified through discussion with our patient and public involvement representatives.

Stakeholder consultation and scoping of the literature will be followed by a workshop where the research team will discuss the findings, begin to identify common concepts and map and prioritise the theory to guide phase 2. This approach has worked well in previous realist reviews ${ }^{52} 53$ and ensures that the focus of the review is relevant and captures complementary and competing accounts. To ensure transparency of approach and an audit trail, we will, with permission, transcribe recordings of group discussions, and maintain structured field notes on suggestions and decision-making processes about which sources of evidence were linked to which strands of theoretical development. ${ }^{32}$ The process will also draw on the existing research, clinical and engagement experiences of the research team.

\section{Phase 2: retrieval, review and synthesis Selection criteria}

Realist synthesis enables the testing of the relevance and rigour of emerging findings from one body of literature to another and, in line with the iterative nature of realist synthesis methodology, ${ }^{54}$ the inclusion criteria will be refined in light of emerging data and the theoretical development in phase 1 . The review is likely to include evidence sources that cover the following:

- community-dwelling older people with complex health and care needs, such as those with frailty, multimorbidity, dementia. The focus will be on those aged 65 and over, although for certain groups (eg, BME, homeless people) younger participants $(\geq 55)$ may be included if the issues are similar;

- older people with complex health needs living in their own homes, in sheltered housing and extra care housing (where people still have the capacity to participate in SDM);

- studies of any intervention or strategy designed to promote the ongoing engagement of older people with complex health needs, and/or their family carers, in decision-making relating to their health or social care needs (eg, decision aids, physician or patient coaching, education or training, personalised care planning and joint goal setting). The focus is on complex decision-making and personal goals rather than studies focused on single issues (such as whether to have a hip operation);

- studies of interprofessional SDM where at least two healthcare professionals collaborate to achieve SDM with the patient and/or family carer either concurrently or sequentially; ${ }^{41}$

- studies that provide evidence relating to the implementation and uptake of interventions designed to promote SDM for older people with complex health needs.

\section{Types of studies}

A diversity of evidence provides an opportunity for richer mining and greater explanation. Therefore, we will include studies of any design including randomised controlled trials, controlled studies, effectiveness studies, uncontrolled studies, interrupted time series studies, cost-effectiveness studies, process evaluations and qualitative studies of participants' views and experiences of interventions. We will also include unpublished and grey literature, policy documents and information about locally implemented programmes in the UK.

\section{Outcomes}

A main aim of the NHS Five Year Forward View ${ }^{6}$ is to tackle the gap between care and quality. Quality is seen in terms of patient safety, clinical effectiveness and patient experience. This definition of quality will be used to guide the outcomes for this review, with a particular focus on the patient experience. However, part of the review process will involve an iterative identification of outcomes that are important to stakeholders, that address patient and family involvement in care planning and decision-making and that have been reported in the literature. Potential outcomes include the following:

A. Patient experience: participation in decision-making, improved match between chosen option and patient preferences, impact on the decision-making experience, quality of life, perceived burden of treatment;

B. Patient safety: access to appropriate care, prevention of adverse events such as falls, avoidable emergency admissions and substantiated abuse and/or neglect; 
C. Clinical effectiveness: health-related outcomes (eg, prevention of exacerbations of long-term conditions), service use (eg, unnecessary hospital admissions, unnecessary GP visits).

\section{Identification of studies}

We will use a range of search techniques including electronic databases and lateral searches. The electronic search strategy will be developed by an experienced information scientist with input from the rest of the project team. We will search the following electronic databases for studies published in English:

- Medline (PubMed), CINAHL, BNI, DH Data, King's Fund, SCOPUS, TRIP, Cochrane Library (incl. CENTRAL, CDSR, DARE, HTA), AgeInfo (Centre for Policy on Ageing-UK).

In addition to the above electronic database searches, we will undertake the following lateral searches:

- checking of reference lists from primary studies and systematic reviews (snowballing) ${ }^{55}$

- citation searches using the 'Cited by' option on Scopus and Google Scholar and the 'Related articles' option on PubMed ('Lateral Searching') ${ }^{56}$

- contact with experts and those with an interest in the care of older people with complex health and social care needs to uncover grey literature (eg, National Library for Health Later Life Specialist Library, Alzheimer's Society, James Lind Alliance).

The search terms used (and number of searches) will be iterative with terms extended and refocused as the review progresses. At this stage, we anticipate that the main search will focus on: (1) interventions to promote SDM with older people with complex needs and (2) interventions involving family carers of older people with complex needs in SDM.

\section{Screening and data extraction}

Electronic search results will be downloaded into bibliographic software and, where identified, duplicates will be deleted. Documents from other sources will be manually recorded in the same file. Two reviewers will independently screen titles and abstracts for relevance. Full manuscripts of all potentially relevant citations will be obtained and downloaded into Mendeley reference management and PDF organisation software. Two reviewers will screen full manuscripts for inclusion based on the relevance and rigour of the evidence, with disagreements resolved by discussion with a third team member. Relevance is defined as the extent to which evidence can contribute to theory building and/or testing, and rigour is defined as the extent to which the methods used to generate that particular piece of data are credible and trustworthy. ${ }^{35} 57$ We will draw on methods used in a previous realist review to create a set of constructs to ensure that the test of rigour and relevance is transparent and clear to all members. ${ }^{58}$ This set of constructs will be added to the data extraction form in the form of a flow chart.
For studies that meet the test of relevance and rigour, data will be extracted onto bespoke data extraction forms which will enable us to collate the evidence on context mechanism and outcomes. ${ }^{34}$ The data extraction form will be informed by programme theories that emerge from phase 1 and will be pretested by the review team on a subset of 5-10 papers. Member checking for data extraction and assessment of relevance and rigour will take place across the core research team; a sample of evidence will be cross-checked across four team members (FB, SD, BR and CG). Once checking has established a good level of agreement, the remaining data will be extracted by one reviewer, with $20 \%$ of records checked by a second reviewer.

\section{Synthesis}

The analytical task is in synthesising the extracted information according to the relationships between mechanisms (eg, underlying processes and structures), contexts (eg, conditions, types of setting, organisational configurations) and outcomes (ie, intended and unintended consequences and impact). Rycroft-Malone et al $(2012)^{34}$ have developed an approach to synthesis, incorporating the work of Pawson (2006) ${ }^{57}$ and principles of realist enquiry, which includes:

1. organisation of extracted information into evidence tables representing the different bodies of literature (eg, decision aids, personalised care planning and joint goal setting, education and training/coaching);

2. theming across the evidence tables in relation to emerging patterns (known as demi-regularities in realist literature) among context, mechanism and outcomes (C-M-Os), seeking confirming and disconfirming evidence;

3. linking these demi-regularities (patterns) to refine hypotheses.

Data synthesis will involve individual reflection and team discussion and will:

(1) question the integrity of each theory, (2) adjudicate between competing theories, (3) consider the same theory in different settings and (4) compare the stated theory with practice experiences. Data from the studies or other evidence will then be used to confirm, refute or refine the candidate theories. Where theories fail to explain the data, alternative theories will be sought.

Once the preliminary mapping of the evidence into tables is complete, the research team will hold a second one-day workshop. This workshop will be structured to include in-depth discussion of the findings and to develop and confirm the resultant hypotheses. These will act as synthesised statements of findings around which a narrative can be developed summarising the nature of the context, mechanism and outcome links, and the characteristics of the evidence underpinning them.

The transparency of a realist synthesis is reliant on careful documentation of the reasoning processes, how they are grounded in the evidence and justification of inferential shifts through engagement with different 
evidence sources. $^{32} 57$ This aspect of the review process is resource intensive and reliant on discussion and deliberation, across and with particular members of the research team.

\section{Phase 3: testing and refining programme theory/theories (validation)}

To enhance the trustworthiness of the resultant hypotheses and develop a final review narrative, we will test the hypotheses and supporting evidence with stakeholders from phase 1 . This will initially be done via email, followed by telephone or face to face interviews with a smaller sample of up to 10 participants purposively selected to reflect the original stakeholder groups. For older people and family carers, the hypotheses will be presented in the form of vignettes which will be developed in conjunction with our patient and public involvement representatives. An interview schedule will be developed based on the findings that have emerged from the synthesis process and will aim to elicit stakeholders' views on their meaningfulness, from practice and service user/carer perspectives. Interviews will be recorded with permission and transcribed.

\section{Dissemination and projected outputs}

Project results will be disseminated via several routes including published reports and papers, stakeholder meetings and engagement with voluntary-sector, and practice organisations. In addition, the professional and research networks of the team, including their strong links to four of the vanguard sites, will be crucial for disseminating findings to the national and international research, practice and policy communities. Knowledge mobilisation will be facilitated through the engagement of stakeholders throughout the conduct of the review. This will include (1) practitioners, managers and commissioners in the vanguard sites and (2) patients, family carers, members of the public, researchers, educators and policymakers with an interest in SDM and person-centred care.

\section{Patient and public involvement}

This project will involve active collaboration with members of the University of Hertfordshire Public Involvement in Research Group (PIRG) and the Social Care Workforce Research Unit's standing User and Carer Group at King's College London. Both groups include members with experience of collaborating on projects relating to the health and care needs of older people and come from a variety of diverse backgrounds. Representatives from these groups will act as members of the advisory group $(\mathrm{n}=2)$, attend project workshops $(n=2)$ and comment on interview transcripts and other project outputs $(n=4)$.

\section{DISCUSSION}

Including the patient perspective is a central organising principle of integrated care. Moreover, there is increasing recognition of the importance of promoting interprofessional approaches to SDM, particularly for individuals most reliant on health and care services, such as the very old and those with long-term conditions. A realist synthesis of the evidence will establish the mechanisms that preserve and foster SDM between providers, patients and carers and how they achieve improvements in patient experiences. By providing possible explanations for the way in which interventions are thought to work and how change is achieved, it will illustrate how to tailor an intervention to new models of care and to this patient group. The propositions arising from the review will also inform the design of future intervention studies.

Author affiliations

${ }^{1}$ Centre for Research in Primary and Community Care, University of Hertfordshire, Hatfield, UK

${ }^{2}$ Social Care Workforce Research Unit, King's College London, London, UK

${ }^{3}$ The Preference Laboratory, The Dartmouth Institute for Health Policy \& Clinical Practice, Lebanon, New Hampshire, USA

${ }^{4}$ Tower Hamlets Clinical Commissioning Group, The Tredegar Practice, London, UK

${ }^{5}$ Research Department of Primary Care and Population Health, UCL Medical School (Royal Free Campus), London, UK

${ }^{6}$ University of Hertfordshire, Hatfield, UK

${ }^{7}$ Centre for Health Service Studies, University of Kent, Canterbury, UK

Twitter Follow Frances Bunn @bunn_f

Contributors FB is responsible for the initial drafting of the manuscript. CG provided contributions on methodology. IH, JM, PM, GR, PW and M-AD provided subject expertise. All authors were involved in the protocol design, critically reviewed the paper and gave final approval of the version to be published.

Funding This article presents independent research commissioned by the National Institute for Health Research (NIHR) under HS\&DR (grant reference number 15/77/25).

Disclaimer The views expressed in this paper are those of the authors and not necessarily those of the NHS, the NIHR or the Department of Health. The sponsor of the study had no role in study design or writing of the paper.

Competing interests All authors have completed the ICMJE uniform disclosure form at http://www.icmje.org/coi_disclosure.pdf and declare: Professor Goodman received personal fees from the National Institute for Health Research (NIHR) during the conduct of the study, all others report no financial relationships with any organisations that might have an interest in the submitted work in the previous 3 years; no other relationships or activities that could appear to have influenced the submitted work.

Ethics approval Approvals have been obtained from the University of Hertfordshire ECDA (Ethics Committee with Delegated Authority), reference number HSK/SF/UH/02387.

Provenance and peer review Not commissioned; externally peer reviewed.

Open Access This is an Open Access article distributed in accordance with the Creative Commons Attribution Non Commercial (CC BY-NC 4.0) license, which permits others to distribute, remix, adapt, build upon this work noncommercially, and license their derivative works on different terms, provided the original work is properly cited and the use is non-commercial. See: http:// creativecommons.org/licenses/by-nc/4.0/

\section{REFERENCES}

1. Jamieson M, Grealish L, Brown J-A, et al. Carers: The navigators of the maze of care for people with dementia-a qualitative study. Dementia 2016;15:1112-23. 
2. Guthrie B, Saultz JW, Freeman GK, et al. Continuity of care matters. BMJ 2008;337:a867.

3. Sansoni JE, Grootemaat PE, Seraji HR, et al. Targeting integrated care to those most likely to need frequent health care: a review of social and clinical risk factors. Sax Institute, Australia, 2015). http:// www.aci.health.nsw.gov.au/_data/assets/ pdf_file/0008/274742/ Evidence_Check_Targeting_integrated_care_Social_and_clinical_ risk_factors.pdf

4. Shaw S, Rosen R, Rumbold B. An overview of integrated care in the NHS. What is integrated care?: Nuffield Trust. 2011. http://www. nuffieldtrust.org.uk/publications/what-integrated-care? gclid=CjOKEQiAiMHEBRC034nx2ImB1JOBEiQA r7ctmz2xMOu4w9f8aJXLWKqiSsO4FYIGIrdvfks OVKUjlaAooz8P8HAQ

5. Natioanal Collaboration for Integrated Care and Support. Integrated care and support: our shared committment. 2013. https://www.gov. uk/government/publications/integrated-care

6. NHS England. Five year forward view. http://www.england.nhs.uk/ wp-content/uploads/2014/10/5yfv-web.pdf. 2014.

7. Goodwin N, Smith J, Davies A, et al. Integrated care for patients and populations: improving outcomes by working together. The Kings Fund, 2011. http://www.kingsfund.org.uk/publications/integrated-carepatients-and-populations-improving-outcomes-working-together (accessed 25 Nov 2014).

8. Dowrick A, Southern A. Dementia 2014: Opportunity for change. In: Society As, ed. London: alzheimers.org.uk/dementia2014 (accessed 21 Mar 2015).2014.

9. Field S. NHS Future Forum: Summary report on proposed changes to the NHS. 2011. http://www.dh.gov.uk/prod_consum_dh/groups/ dh_digitalassets/documents/digitalasset/dh_127540.pdf (accessed on 22nd April 2015).

10. Coulter A, Collins A. Making shared decision-making a reality No decision about me, without me. The Kings Fund, 2011

11. Charles C, Gafni A, Whelan T. Shared decision-making in the medical encounter: what does it mean? (or it takes at least two to tango). Soc Sci Med 1997;44:681-92.

12. DH. Equity and excellence: liberating the NHS. 2010. https://www. gov.uk/government/uploads/system/uploads/attachment_data/file/ 213823/dh 117794.pdf.

13. Stacey D, Légaré F, Col Nananda F, et al. Decision aids for people facing health treatment or screening decisions. Cochrane Database Syst Rev 2014;(1):CD001431. http://onlinelibrary.wiley.com/doi/10. 1002/14651858.CD001431.pub4/abstract.

14. Da Silva D. Helping people share decision making a review of evidence considering whether shared decision making is worthwhile. The Health Foundation, 2012.

15. Elwyn G, Scholl I, Tietbohl C, et al. "Many miles to go..." a systematic review of the implementation of patient decision support interventions into routine clinical practice. BMC Med Inform Decis Mak 2013:13(Suppl 2):S14.

16. Banerjee S. Multimorbidity-older adults need health care that can count past one. Lancet 2014;385(9968):587-9.

17. Bunn F, Goodman C, Brayne C, et al. Comorbidity and dementia: a mixed-method study on improving health care for people with dementia (CoDem). Health services and delivery research 2016;4 (8): http://www.journalslibrary.nihr.ac.uk/_data/assets/pdf_file/0018/ 162216/FullReport-hsdr04080.pdf.

18. Leppin A, Montori V, Gionfriddo M. Minimally disruptive medicine: a pragmatically comprehensive model for delivering care to patients with multiple chronic conditions. Healthcare 2015;3:50-63.

19. Gagnon LM, Patten SB. Major depression and its association with long-term medical conditions. Can J Psychiatry 2002;47: $149-52$.

20. Freeman GK, Woloshynowych M, Baker R, et al. Continuity of care 2006: What have we learned since 2000 and what are policy imperatives now. London: National Coordinating Center for NHS Service Delivery and Organisation R \& D (NCCSDO), 2007.

21. Bunn F, Burn AM, Goodman C, et al. Comorbidity and dementia: a scoping review of the literature. BMC Med 2014;12:192.

22. Dixon-Woods M, Cavers D, Agarwal S, et al. Conducting a critical interpretive synthesis of the literature on access to healthcare by vulnerable groups. BMC Med Res Methodol 2006;6:35.

23. Frampton S, Guastello S, Brady C, et al. Patient-centered care: Improvement guide. Derby, CT and Camden, ME: Planetree, Inc/ Picker Institute, 2008. http://planetree.org/Centered-CareImprovement-Guide-10-28-09-Final.pdf (accessed 24 Nov 2014).

24. Hannan R, Thompson R, Worthington $A$, et al. The triangle of care. Carers included: a guide to best practice for dementia care. Carers Trust, 2013. http://www.rcn.org.uk/_data/assets/pdf_file/0009/ 549063/Triangle_of_Care_-_Carers_Included_Sept_2013.pdf (accessed 25 Nov 2014)
25. Johnson B, Abraham M, Conway J, et al. Partnering with patients and families to design a patient- and family- centered health care system. Institute for Patient- and Family-Centered Care and Institute for Healthcare Improvement. 2008. http://www.ihi.org/resources/ Pages/Publications/Partneringwith-PatientsandFamilies.aspx (accessed 22 Apr 2015).

26. Sinclair A, Morley JE, Rodriguez-Manas L, et al. Diabetes mellitus in older people: position statement on behalf of the International Association of Gerontology and Geriatrics (IAGG), the European Diabetes Working Party for Older People (EDWPOP), and the International Task Force of Experts in Diabetes. J Am Med Dir Assoc 2012;13:497-502.

27. Sinclair AJ, Hillson R, Bayer AJ, et al. Diabetes and dementia in older people: a best clinical practice statement by a multidisciplinary National Expert Working Group. Diabet Med 2014;31:1024-31.

28. Demain S, Goncalves AC, Areia C, et al. Living with, managing and minimising treatment burden in long term conditions: a systematic review of qualitative research. PLOS ONE 2015;10:e0125457.

29. May C, Montori VM, Mair FS. We need minimally disruptive medicine. BMJ 2009;339:b2803.

30. Ridgeway JL, Egginton JS, Tiedje K, et al. Factors that lessen the burden of treatment in complex patients with chronic conditions: a qualitative study. Patient Prefer Adherence 2014;8: 339-51.

31. Goodman C, Drennan V, Manthorpe J, et al. A study of the effectiveness of inter professional working for community dwelling older people Final Report. In: NIHR Service, Delivery, and, organisation, programme. ed. 2011.

32. Hardwick R, Pearson M, Byng R, et al. The effectiveness and cost-effectiveness of shared care: protocol for a realist review. Syst Rev 2013;2:12.

33. Pawson R, Greenhalgh T, Harvey G, et al. Realist review--a new method of systematic review designed for complex policy interventions. J Health Serv Res Policy 2005;10(Suppl 1) 21-34.

34. Rycroft-Malone J, McCormack B, Hutchinson AM, et al. Realist synthesis: illustrating the method for implementation research. Implement Sci 2012;7:33.

35. Wong G, Greenhalgh T, Westhorp $\mathrm{G}$, et al. RAMESES publication standards: realist syntheses. BMC Med 2013;11:21.

36. Kastner M, Estey E, Perrier L, et al. Understanding the relationship between the perceived characteristics of clinical practice guidelines and their uptake: protocol for a realist review. Implement Sci 2011;6:1-9.

37. Elwyn G, Edwards A, Kinnersley P, et al. Shared decision-making and the concept of equipoise: the competences of involving patients in healthcare choices. Br J Gen Pract 2000;50:892-9.

38. Barry MJ, Edgman-Levitan S. Shared decision making-the pinnacle of patient-centered care. N Engl J Med 2012;366:780-1.

39. Makoul G, Clayman ML. An integrative model of shared decision making in medical encounters. Patient Educ Couns 2006;60:301-12.

40. Légaré F, Stacey D, Gagnon S, et al. Validating a conceptual model for an inter-professional approach to shared decision making: a mixed methods study. J Eval Clin Pract 2011;17: 554-64.

41. Légaré $\mathrm{F}$, Stacey $\mathrm{D}$, Pouliot $\mathrm{S}$, et al. Interprofessionalism and shared decision-making in primary care: a stepwise approach towards a new model. $J$ Interprof Care 2011;25:18-25.

42. Durand MA, Carpenter $\mathrm{L}$, Dolan $\mathrm{H}$, et al. Do interventions designed to support shared decision-making reduce health inequalities? A systematic review and meta-analysis. PLOS ONE 2014;9:e94670.

43. Pearce C, Bainbridge M. A personally controlled electronic health record for Australia. J Am Med Inform Assoc 2014;21:707-13.

44. Farrelly S, Brown Gill E, Flach C, et al. User-held personalised information for routine care of people with severe mental illness. Cochrane Database Syst Rev 2013;(10):CD001711. http:// onlinelibrary.wiley.com/doi/10.1002/14651858.CD001711.pub2/ abstract

45. Detmer D, Bloomrosen M, Raymond B, et al. Integrated personal health records: transformative tools for consumer-centric care. $B M C$ Med Inform Decis Mak 2008;8:45.

46. Wilson PM, Kendall S, Brooks F. The Expert Patients Programme: a paradox of patient empowerment and medical dominance. Health Soc Care Community 2007:15:426-38.

47. Wilson PM, Kendall S, Brooks F. Nurses' responses to expert patients: the rhetoric and reality of self-management in long-term conditions: a grounded theory study. Int J Nurs Stud 2006;43:803-18. 
48. Johnson M, Tod AM, Brummell S, et al. Prognostic communication in cancer: a critical interpretive synthesis of the literature. Eur J Oncol Nurs 2015;19:554-67.

49. Bélanger E, Rodríguez C, Groleau D. Shared decision-making in palliative care: a systematic mixed studies review using narrative synthesis. Palliat Med 2011;25:242-61.

50. Freeman G. Hughes J. Continuity of care and the patient experience. London: The Kings Fund, 2010. http://www.kingsfund.org.uk/sites/files/ $\mathrm{kf} /$ /ield/field_document/continuity-care-patient-experience-gp-inquiryresearch-paper-mar11.pdf (accessed 16 Apr 2015).

51. Parker G, Corden A, Heaton J. Experiences of and influences on continuity of care for service users and carers: synthesis of evidence from a research programme. Health Soc Care Community 2011;19:576-601.

52. Goodman C, Rycroft Malone J, Norton C, et al. Reducing and managing faecal incontinence in people with advanced dementia who are resident in care homes: protocol for a realist synthesis. BMJ open 2015;5:e007728.
53. Bunn F, Goodman C, Trivedi D, et al. Managing diabetes in people with dementia. A realist synthesis: NIHR 13/138, 2015.

54. Pawson R, Greenhalgh T, Harvey G, et al. Realist synthesis: an introduction. ESRC Research Methods Programme. Manchester: University of Manchester, 2004.

55. Dixon-Woods M. Searching for studies in complex areas. http:// wwwccsracuk/methods/festival/programme/rsy2/documents/ dixon-woodsppt 2006.

56. Greenhalgh T, Peacock R. Effectiveness and efficiency of search methods in systematic reviews of complex evidence: audit of primary sources. BMJ 2005;331:1064-5.

57. Pawson R. Evidence-based policy: a realist perspective. Sage publications, 2006.

58. Rycroft-Malone J, Burton CR, Williams L, et al. Improving skills and care standards in the support workforce for older people: a realist synthesis of workforce development interventions. Health Ser Deliv Res 2016;4:37-48. 